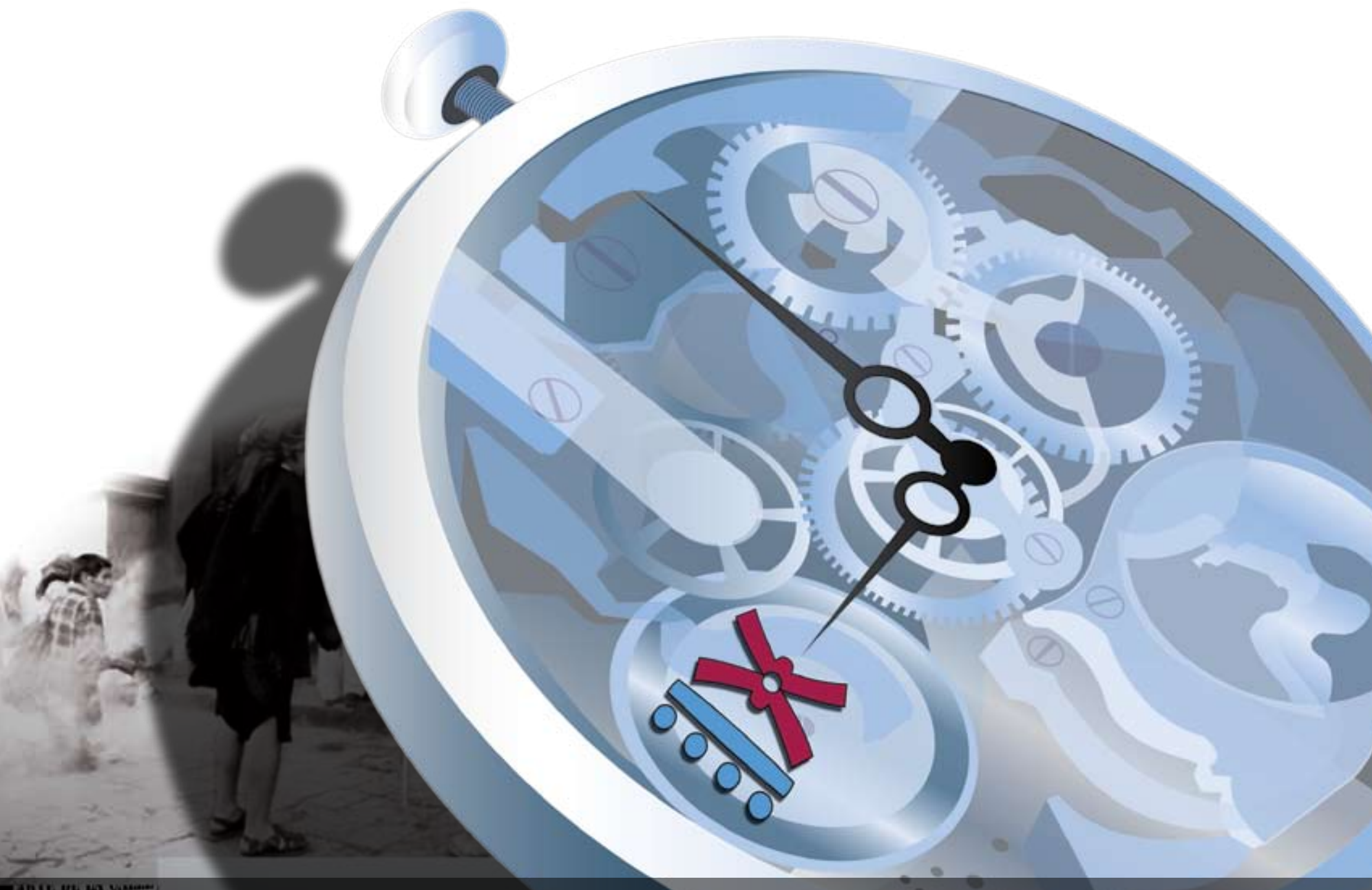

$\lambda 9^{\circ}$ C O N RESO 2. CENTROAMERICANO DE H IS T O R I A

ISSN 1409-469X

Fecha de recepción: 15 de mayo 2008 Fecha de aceptación: 30 de mayo 2008

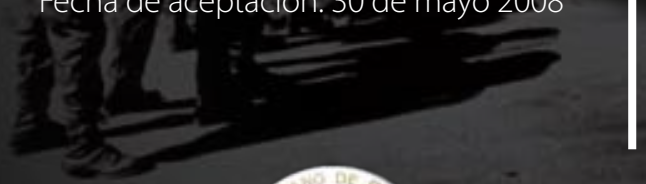

Las identidades de la población de origen jamaiquino en el Caribe costarricense, 1872-1950

Miembros del Consejo Editorial:

Dr. Ronny Viales, Dr. Juan José Marín

Editores Técnicos:

Allan Fonseca, Andrés Cruz, Gabriela Soto
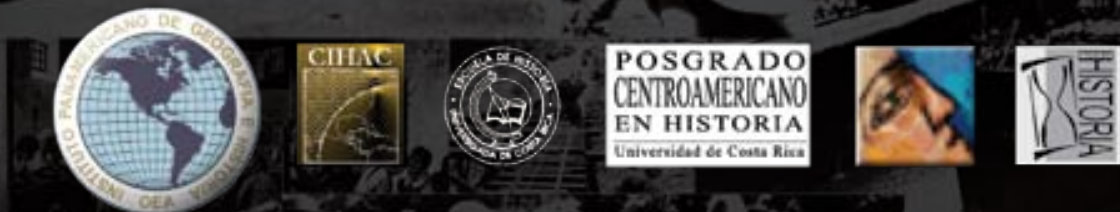
Indexaciones: Repositorio de Revistas UCR, DIALNET, Latindex, REDALYC Directorio y recolector de recursos digitales del Ministerio de Cultura de España, Directory of Open Access Journals. Diálogos Revista Electrónica de Historia ISSN 1409- 469X. Número especial 2008. Dirección web: http://historia.fcs.ucr.ac.cr/dialogos.htm

\title{
Las identidades de la población de origen jamaiquino en el Caribe costarricense, $1872-1950$
}

\author{
Reina Cristina Rosario Fernández
}

Universidad de Costa Rica, UCR. Tres Ríos, Costa Rica, Tel. 8346-8070

reinacrf@gmail.com 
Este trabajo forma parte del primer capítulo de la tesis titulada: "Las identidades de la población de origen jamaiquino en el Caribe costarricense, 1950-2000”. Su objetivo es analizar el proceso sociohistórico vivido por la población de origen jamaiquino del Caribe costarricense en el periodo 1872-1950, a fin de identificar cuáles fueron los elementos aglutinadores de las primeras formas de identidades étnicas elaboradas por esta población.

Para lograr este objetivo, se responderá a la pregunta: ¿Cuáles fueron las primeras manifestaciones de identidad étnica de los inmigrantes jamaiquinos en el Caribe costarricense, en el marco de las políticas estatales en el periodo 1872-1950? La respuesta se obtiene de periódicos de la época, documentos oficiales y de diversas organizaciones de la comunidad; además, se usa con discreción la historia oral, para conocer cómo ve su pasado la comunidad de hoy; y también se recurre a la historiografía existente.

En la construcción de las identidades de la población estudiada, intervienen varios actores fundamentales. En este estudio se analiza el rol del Estado, el de la compañía bananera, el de la población no negra y el de la población negra, como actores colectivos.

\section{Introducción}

La región caribeña de Costa Rica se diferencia del resto del país, no solo por sus características naturales: geografía, flora, fauna y clima, sino también por la historia. En el periodo colonial, resultó imposible la conquista de estos territorios debido a la ferocidad con que los defendían los indígenas que la habitaban. Por ello esta región se convirtió en un sitio de frontera, sin población, lo cual la mantuvo aislada del resto del país.

En la etapa colonial, así como en la republicana, se subestimó o marginó la integración de esta región al resto del país. Hubo un gran vacío político y una economía de subsistencia desarrollada por los indígenas de Talamanca. Atendiendo a esta marginación, en el Valle Central se va formando la idea del Caribe como una zona de pobladores diferentes, con lenguas y culturas extrañas.

Ya para el proceso de la invención de la nación costarricense, esta zona no formaba parte del imaginario nacional. Dado este aislamiento, se comprende por qué la región ha tenido su propia historia, con características económicas y culturales muy particulares que la distinguen significativamente del resto del país. 
El Estado se fija en la región del Caribe a fines del siglo XIX, con el fin de comercializar la producción cafetalera del Valle Central hacia Europa. Concibió la idea de construir el ferrocarril en el Caribe para reducir los costos del transporte. El presidente Tomás Guardia firmó en 1871 un contrato con el empresario Henry Keith para construirlo. En 1883 se firma el contrato Soto-Keith, el cual implicó la pérdida del control nacional del ferrocarril, la fecha simbólica del nacimiento del imperialismo en Costa Rica ${ }^{1}$ y el inicio de la producción bananera para exportación, que luego se convertiría en enclave bananero.

Con la entrada del capital imperialista a Costa Rica, no solo se modifica por completo la economía de la región, sino también la composición etnocultural. Llegan inmigrantes, alemanes, belgas, suizos e ingleses, pero los costos económicos obligaron a contratar a chinos, jamaiquinos, curazaleños, italianos, culíes, y centroamericanos, entre otros. La mezcla interétnica ha producido mulatos, mestizos, zambos, y esto ha configurado un compuesto sociocultural que le confiere un colorido único a la región caribeña.

Aunque Costa Rica tiene una costa caribeña de $112 \mathrm{Km}$., estuvo aislada de los procesos regionales hasta finales del siglo XIX. En esta época comienza la entrada en la región de los capitales norteamericanos, con la economía de enclave² (dependencia económica en todo el Gran Caribe) y la inmigración masiva jamaiquina. De esta forma, la región caribeña de Costa Rica se une económica y culturalmente al Gran Caribe.

\section{Ferrocarril, compañía bananera y flujos migratorios}

La llegada de los jamaiquinos a Costa Rica, en 1872, está asociada a la construcción del ferrocarril y al inicio de la economía de enclave bananero en la región del Caribe. Por una parte, estas obras demandaban una abundante mano de obra que la población local no podía suplir. Por otro lado, varias islas del Caribe, y en especial Jamaica, enfrentaban una fuerte crisis económica, por lo cual los caribeños buscaban trabajo fuera de sus fronteras.

1 Jussi Pakkasvirta y Kent Wilsa (editores), “El Caribe centroamericano (Helsinki, Finlandia: Instituto Renvall 18, 2005), 14. El primer contrato (1871) entre el Gobierno de Costa Rica y la dinastía Meiggs-Keith se hizo con el mismo Herny Meiggs, quien traspasó su contrato a su sobrino Henry Keith. El hermano menor de este, Minor Cooper Keith, llegó a entender lo ventajoso que sería el contrato del ferrocarril si se coordinaba con el negocio del banano. Véase: Watt Stevart. “Leith y Costa Rica” (Editorial Costa Rica, San José, 1991 ), 32.

2 Desde fines del siglo XIX, el imperialismo norteamericano se propuso dominar la zona del Caribe, por ser la llave a dos grandes océanos: el Atlántico y el Pacífico. Estados Unidos considera estos dos océanos como sus fronteras. La importancia estratégica del Caribe para esa nación se analiza en el libro del escritor dominicano Juan Bosch, De Cristóbal Colón a Fidel Castro: el Caribe, frontera imperial, Casa de las Américas, 1981. 
R. Harpelle estima que entre 1850 y 1950 llegaron a Mesoamérica unos 400000 descendientes de africanos. ${ }^{3}$ Con respecto a la inmigración a Costa Rica, considera que entre 1891 y 1911 vinieron 43438 jamaicanos. $^{4}$

Las condiciones de vida y trabajo en el Caribe costarricense fueron duras y peligrosas, en particular en los primeros años, cuando no había viviendas, transportes ni agua potable, y el área era inhóspita. Según varias fuentes documentales, se convirtió en un problema encontrar trabajadores que se adaptaran a la zona y al duro trabajo. ${ }^{5}$ Se ensayó con italianos, holandeses, canadienses, sirios, turcos, negros estadounidenses y antillanos. ${ }^{6}$ Llegaron tanto del Caribe insular como continental. Si bien hubo un indiscutible predominio de los jamaiquinos, también vinieron desde Nueva Orleans, Belice, la costa Caribe de Guatemala, Honduras, Nicaragua, Panamá, Cartagena y Surinám, y de Aruba, Curazao, Saint Thomas, Saint Kitts, Trinidad, Barbados, Martinica y Guadalupe. ${ }^{7}$

La búsqueda cesó cuando en la última década del siglo XIX la Compañía presumió que los jamaiquinos eran los mejores para explotar. Así, empezaron a llegar con regularidad hasta la década de 1920; ya en 1927 había en Limón 19136 jamaiquinos. ${ }^{8}$ No todos los que querían trabajar podían hacerlo; debían pasar pruebas de reclutamiento, incluyendo un riguroso examen físico, el cual pasaban unos veinte de cada cien. ${ }^{9}$ Los seleccionados, jóvenes fuertes y sanos,

$3 \quad$ Harpelle, 14.

$4 \quad$ Ibíd., 17. Otros estudiosos del tema ofrecen cifras diferentes. A. Chomsky sostiene que entre 1900 y 1943 pudo contabilizar un total de 23443 individuos. Véase: Chomsky, 44-45. Por su parte, para Bourgois, Keith aportó 10000 negros de Jamaica entre 1881 y 1891. Véase: Bourgois, 90. Como se nota, la diferencia entre los datos de Harpelle y Bourgois es grande. Por nuestra parte, interesa mostrar la diversidad de datos sobre el volumen de estos inmigrantes, pues por lo regular los datos usados por los historiadores son oficiales o de periódicos y no se registran las inmigraciones no oficiales, que aumentarían estas cifras significativamente.

$5 \quad$ Carmen Murillo, Philippe Bourggois; Quince Duncan; Olien Michael, entre otros.

6 Rodríguez Bolaños, José y Víctor Borge Carvajal. El ferrocarril al Atlántico en Costa Rica. Tesis de licenciatura UCR, Depto. de Sociología, 1976, 52. Dos grupos asociados a la construcción del ferrocarril emigraron del país sin dejar huellas profundas; los italianos, que después de la huelga de 1888 salen del país, y los pocos que quedan se van a Cartago; los coolíes, mezcla de indú y negro, que en su mayoría abandonan el país o se mezclan con negros. Véase: Municipalidad de Limón, cien años de luchas y esperanzas, 1992, 47.

$7 \quad$ No se cuenta con registros precisos para cuantificar esta migración, principalmente por las vías informales en que se desarrolló y por las constantes entradas y salidas de la población a través de los diferentes puntos de embarque. Véase: Murillo Carmen, "Vaivén de arraigos de desarraigos: identidad afro-caribeña en Costa Rica. 1870-1940”. Revista de Historia (Costa Rica) 39 (enero-julio 1999): 186.

8 Olien, Michael. The Negro in Costa Rica: The Etnohistoty of an Ethnic Minority in a Complex Society. Tesis. Departament of Sociology (University of Oregon. 1967), 126.

$9 \quad$ Bourgois, 92. 
morían por la explotación a la que se sometían, sin contratos escritos, víctimas de maltrato y de atrasos del pago. Unos 4000 jamaiquinos murieron en las primeras veinticinco millas del ferrocarril. ${ }^{10}$ Los sueños de ahorrar y volver a su patria se frustraron al hallar en estas tierras solo penalidades.

En la construcción de las identidades de la población en estudio, participan diversos actores, colectivos e individuales, políticos y económicos. En este trabajo se analiza al Estado como actor político y público, a la compañía bananera como actor económico y privado, y como actores colectivos a la comunidad no negra y a la comunidad negra de origen jamaiquino.

\section{El Estado costarricense como actor político y público}

Antes de la llegada de los inmigrantes jamaiquinos, el Estado liberal costarricense, interesado en colonizar nuevos territorios, asumió en parte el modelo racista argentino de "poblar es civilizar”. Cabe recordar que en nuestro continente, en el siglo XIX, con el surgimiento de las constituciones después de las guerras de independencia, no se tomaron en cuenta los indígenas ni los negros, por lo cual no se consideraron ciudadanos. En el caso costarricense, además de negarles tal condición, se ha tratado de invisibilizarlos. ${ }^{11}$

La ley fundamental que regula la inmigración a territorio costarricense, data de 1862 y se llama Ley de Bases y Colonización. En su artículo 1, inciso 3, estipula: "No se permitirá la colonización de "razas africanas y chinas" y, en caso de que esta inmigración se considere imprescindible, el gobierno queda facultado para limitarla y controlarla”. ${ }^{2}$

Pero fueron los negros jamaiquinos los elegidos para el difícil trabajo en la región. Meléndez y Duncan señalan sobre este periodo: "El Estado levantó la prohibición de la inmigración negra y china, decretada en 1862, porque pensaba que una vez concluida la obra del ferrocarril, estos inmigrantes ocasionales saldrían hacia otra parte". ${ }^{13}$ Pero la crisis económica del ferrocarril se sentía venir desde 1874, y obligó a muchos inmigrantes a dedicarse a la agricultura de subsistencia, por lo cual permanecían en la región ${ }^{14}$.

10 Thomas Deborad, Nacionalism and Globalization, and the Politics of Culture in Jamaica, citado por Ronald Soto Quirós, 8.

11 David Díaz Arias, “La invención de las naciones en Centroamérica”. Ponencia presentada en el Coloquio “Identidad” (Instituto de Historia en Nicaragua y C. A., 28 y 29 de octubre de 2004), 22.

ANCR. Serie Gobernación.23426. Folio 48.1850.

Para evitar la salida de trabajadores que se pueden ocupar en la empresa, desde el Estado se instruyó 
Como se indicó antes, el Estado, con el fin de abaratar el transporte del café hacia Europa, firma en 1871 un contrato con el empresario Henry Meiggs Keith para construir una línea ferroviaria entre San José y el puerto de Limón. Por los problemas económicos en la construcción del ferrocarril, el 13 de julio de 1883 se firma el contrato Soto-Keith, el cual significó la pérdida de control nacional del ferrocarril.

A cambio del refinanciamiento de la deuda externa de Costa Rica y la construcción de un tramo de $84 \mathrm{~km}$ de vía férrea, el contrato cedía a Minor C. Keith el arrendamiento por 99 años de $148 \mathrm{~km}$ de ferrocarril construidos; la propiedad de 800000 acres ingleses de tierra virgen, situados a ambos lados de la línea o en otros sitios del país; la exención de impuestos para importar materiales de construcción y la exención para las tierras cedidas, durante veinte años. El Congreso aprobó el contrato en abril de $1884 .^{15}$

El Estado costarricense entregó la zona caribeña al enclave bananero y la abandonó a su suerte. La Compañía se encargaba de la salud, construcción de caminos y otros servicios básicos, y las construcciones y servicios hechos por el Estado fueron escasos. No fue hasta 1945 cuando el Gobierno creó ${ }^{16}$ el Colegio de Segunda Enseñanza, que empezó labores en marzo de 1946. En estas escuelas, al inicio predominaban alumnos negros, pero para la década de los cincuentas solo conformaban el 50\% del alumnado, lo que las convierte en un espacio de socialización con los demás grupos étnicos de la región.

En medio de la crisis de 1930, el Estado cambió su política de inmigración de los años veintes e instauró estrictos controles sobre los trabajadores extranjeros, como la penalización a quienes ingresaran sin efectuar los trámites. Se creó el "Registro de identificación inmigratoria", y en 1930 se aplicaron fuertes controles burocráticos y policíacos sobre los extranjeros. Según el artículo 8 de la Ley de Registro, el extranjero que ingrese en el país clandestinamente, deberá salir de inmediato. ${ }^{17}$ Esta ley diferencia entre "extranjeros distinguidos”, que son bien recibidos, $\mathrm{y}$ “extranjeros sospechosos”, que aun cuando se acepten, deben mantenerse bajo estricto control. para dar a cada trabajador que se fincara al lado de la línea entre Limón y el Campamento n. ${ }^{\circ} 2$, un terreno de 50x50 varas, cuyo título se le daría cuando llegara el caso. Véase: Gaceta Oficial, 21 de marzo de 1874, 1.

15 Ronald Soto Quirós, "Un otro significante en la identidad nacional costarricense: el caso del inmigrante caribeño, 1972-1926” ( Boletín No.25 AFIHC Fassac, France) (octubre 2006), 8

16 Gaceta Oficial n. ${ }^{\circ} 289$, del 23 de diciembre de 1945. Creación del colegio de segunda enseñanza.

17 "Registro de identificación inmigratoria", en Leyes y decretos, 3 de setiembre 1930, citado por Alvarenga Venutulo, Patricia, "La inmigración extranjera y la nación costarricense”. Encuentro Internacional "Poblaciones migrantes y derechos humanos en América Latina” (Heredia, Costa Rica: UNA, 2005, 14. 
En la ley n. ${ }^{\circ} 39$ de 1931, se señala que todo inmigrante debe presentar mil colones en efectivo. El artículo 2 autoriza al Estado a "rechazar... a aquellos inmigrantes que considere nocivos para el país". ${ }^{18}$

En marzo de 1933, en vista del vencimiento de la ley n. ${ }^{\circ}$ 39, el Estado crea un nuevo instrumento jurídico para, además de exigir pagos en efectivo, controlar a las inmigraciones pasajeras que por especulación visiten al país con espectáculos. ${ }^{19}$ Se establece entonces la vigencia de la ley 39 y, aparte de la presentación de los mil colones, el depósito de esta suma. ${ }^{20}$ También, en 1933 se restringe la participación de extranjeros en asuntos nacionales, el Congreso derogó la ley que permitía a los extranjeros ser munícipes. ${ }^{21}$

Paralelo a estos controles, y ante la imposibilidad de traer inmigrantes europeos, el Gobierno fomentó la "auto-inmigración”22, creó la colonia agrícola de Guápiles y ofreció a los colonos condiciones muy atractivas, como títulos de tierras y facilidades agrícolas.

En 1934, una nueva ley evidenciaba el criterio discriminatorio y racista del Estado. Ante el traslado de la compañía bananera del Caribe al Pacífico Sur, el 10 de diciembre se dictó la ley cuyo artículo 5 indica: "Queda prohibido en la zona del Pacífico, ocupar gente de color en dichos trabajos (de producción y explotación bananera)". ${ }^{23}$ Además de esta ley, existe en la memoria de la comunidad limonense y de los costarricenses en general, una ley que prohibía a los negros pasar de Turrialba. Si bien la búsqueda de tal prohibición ha sido infructuosa para los estudiosos

18 Decreto n. ${ }^{\circ}$ 39.5/3/1931, ANCR. Serie Congreso n. ${ }^{\circ}$ 16018, f.9, citado por Ronald Soto, "Discursos y políticas de inmigración en Costa Rica: 1862-1943”, revista Iberoamericana. Madrid, España) 19 (2005): 126.

19 Proyecto de ley. 14/3/1933 ANCR. Serie Congreso. No.16297, f.1.

20 Ibíd.

21 Soto Quirós, “ Discursos...”, 126.

$22 \mathrm{Al}$ inicio del siglo, el Estado muestra interés en seleccionar inmigrantes para vigorizar la raza en términos de regeneración o procurar la "auto-inmigración", que significaba priorizar el saneamiento, la higiene para evitar los altos índices de mortalidad y conservar el bienestar biológico de la raza nacional. Cleto González Víquez insistía en que era más conveniente mejorar las condiciones de salubridad de los habitantes para que la población tuviera un rápido crecimiento natural, que traer inmigrantes. Steven Palmer apunta que González Víquez denominaría a esta política la "auto-inmigración”: llevar al máximo la producción y la reproducción nacional..." Véase: Palmer S. "Hacia la auto-inmigración”. El nacionalismo oficial de Costa Rica", p. 113. En la Memoria de Gobernación y Fomento de 1905 y 1906, las autoridades se refieren al problema de la alta mortalidad infantil y señalan la ignorancia materna como una de sus principales causas, pues a menudo las mujeres, en lugar de acudir al médico, visitan al curandero: “...con tales hábitos profundamente arraigados en las costumbres populares... ¿¿cómo será posible esperar que Costa Rica Crezca Fecunda por sí misma”.

23 Gaceta Oficial, 1935, Ley n. ${ }^{\circ} 31$. 
del tema, lo cierto es que se ejecutaba. ${ }^{24}$

En el discurso oficial, la inmigración negra aparece claramente como un "problema" que debe tolerarse en aras del desarrollo de los trabajos del ferrocarril y, posteriormente, de la compañía bananera. Pero el Estado, lejos de proponer la "integración” del Caribe con el "universo costarricense”, creó una región como "excepción”. Según Murillo, el Caribe fue concebido "como tierra con pobladores diferentes, de lenguas y costumbres "extrañas" con leyes y actividades distintas...”. ${ }^{25}$ El Estado siguió una línea discriminatoria, ampliada en el Gobierno de Calderón Guardia en 1942 al explicitar en el Reglamento de Inmigración el impedimento de determinadas personas: "Personas inconvenientes, nocivas o peligrosas al orden o progreso de la República o a la conservación de la raza, ya sea por sus tendencias agitadoras, ya por sus escasos medios de subsistencias o por las características que predominen en ellas y sean de notoria desafinidad con la población nacional”. ${ }^{26}$

Esa categoría de desafinidad abarcaba a todas las personas no blancas, en especial los negros. Junto a estas leyes de restricciones a determinados pueblos, se estimulaba la inmigración selectiva de europeos; pero estos proyectos (con excepción de la italiana a San Vito) fueron fallidos. ${ }^{27}$ En el siglo XIX y la primera mitad del siglo XX, con estas leyes el Estado estimuló la inmigración de europeos, exaltando el carácter de excepcionalidad de Costa Rica como pacífica y blanca. Estos discursos crearon una corriente xenófoba contra la población negra, que por mucho tiempo impidió a los descendientes de jamaiquinos nacidos en el país, obtener la ciudadanía, y por ello debían optar por la naturalización.

La relación del Estado costarricense con la población negra del Caribe costarricense, se sintetiza en la memoria colectiva de la población negra de hoy de la siguiente forma:

“Al principio está claro que hubo una gran dosis de racismo y discriminación, era la

$24 \quad$ Bourgois relata que costarricenses le contaron que durante la Segunda Guerra Mundial se prohibió a los negros visitar el resto del país. Lo esencial de esta creencia demuestra que, aun si la restricción a los viajes de los negros fuera de la provincia Atlántica no se codificó, existió una gran hostilidad hacia ellos fuera de la región hacia los años cincuentas, pues de otra manera el mito no estaría tan arraigado, Véase: Bourgois, 143.

25 Murillo Chavarri, Carmen, "Identidades de hierro y humo. La construcción del ferrocarril del Atlántico.1879-11890” (San José: Editorial Porvenir, 1995).

26 Soto Quirós, "Inmigración e identidad nacional en Costa Rica 1904-1942. Los "otros" reafirman el “nosotros”. Tesis para optar por el grado de Licenciatura en Historia (San José, UCR. 1998), 257.

27 Alveranga, 10 "La inmigración extranjera y la nación costarricense”. Muestra cronológicamente los intentos del Estado por traer europeos: alemanes, españoles, italianos; y la creación de una legislación que tiende a hacer cada vez más restrictiva la inmigración no deseada, en especial la de chinos y negros. 
tendencia mundial. No fueron tratados como costarricenses hasta mucho tiempo después de haber estado en Costa Rica. Se les rechazaba su cultura y sus características físicas. Lo cual no era exclusivo de Costa Rica, no exclusivo hacia las poblaciones negras. Está claro que al principio, en muchos casos tiende a haber un rechazo hacia las poblaciones de inmigrantes. Sin embargo ese rechazo fue mutuo. La población afro-costarricense durante mucho tiempo no tuvo ningún interés en ser costarricense. Ellos se consideraron superiores al ser súbditos de la Corona Inglesa. Para los jamaicanos, los costarricenses eran gente mal educada y con hábitos no muy limpios. Esto influyó también en la creación de sus propias escuelas y grupos sociales. Ellos querían regresar a Jamaica”. ${ }^{28}$

Esta cita refleja parte de la memoria histórica de la comunidad negra de hoy, sobre la limitada relación del Estado con la comunidad negra en las primeras décadas de la inmigración. Los jamaiquinos se sentían superiores a los costarricenses, y estos a su vez, por el simple hecho de considerarse blancos, se creían superiores a los jamaiquinos. En la apreciación de su pasado, se nota el orgullo de la comunidad negra en definirse como súbditos británicos, y su poco interés por integrarse a la nación que los rechazaba. Sin embargo, M. Olien ${ }^{29}$ plantea que, a finales de los años cuarentas, el registro masivo de negros para hacerse ciudadanos al revocarse las leyes que impedían la ciudadanía, muestran que la adhesión militante a la identidad británica pudo haber sido una forma de defensa ante la discriminación.

Desde las primeras décadas de la inmigración, con frecuencia se etiquetaban a los diferentes grupos de inmigrantes, y la prensa y los demás medios de comunicación ayudaban a crear dichas etiquetas. Mientras los chinos eran relacionados con fraude, vicio y enfermedad, a los jamaiquinos los asociaban con violencia y muerte; los espacios noticiosos los vinculaban con atentados, crímenes e incendios, así como con rituales y prácticas ajenas a los costarricenses, como la pocomía. ${ }^{30}$

28 Entrevista efectuada por internet a Shirley Campbell Barr, residente en Jamaica; 16 de noviembre de 2006.

29 Olien, Michael D. The Negro in Costa Rica: The Ethnohistory of an Ethnic Minority in a Complex Society. Ph.D. Dissertation. University of Oregon, 1967, 148.

30 Según R. Harpelle, los precursores de lo conocido como pocomía en 1930, habían llegado a Costa Rica con los inmigrantes a finales del siglo XIX. Véase: Harpelle, 105. Por su parte, Chomsky refiere la importancia de las tradiciones religiosas afrocaribeñas presentes en las huelgas de 1910, e indica que dos de las principales tradiciones religiosas de Jamaica, de base africana, en el siglo XVII fueron el mialismo y el Obeah. Pocomía constituía la versión más reciente de la religión africana en Jamaica y se basa en danza, tambores, canto y 
Otro elemento importante señalado por varios estudiosos ${ }^{31}$ del tema, es la falta de unidad entre los líderes negros. A menudo se adhirieron a autoridades del Gobierno y colaboraron con la Compañía para consolidar sus propios intereses, en detrimento de la comunidad. Algunos líderes, por congraciarse con la Compañía o el Estado, le restan importancia al racismo existente y justifican situaciones raciales con problemas sociales.

Es importante indicar que la situación de "residente temporal" de los descendientes jamaiquinos nacidos en el país, tuvo un papel significativo en el contexto histórico y social; por un lado, impedía su participación política como ciudadanos y, por otro, después de la Ley del 1934 les prohibía trabajar en el Pacífico Sur, lo cual los mantenía en un confinamiento geográfico.

A principios de siglo XX, pocos se animaron a enfrentar los trámites burocráticos para la naturalización por opciones..$^{32}$ La Secretaría de Relaciones Exteriores publicó que desde 1829 hasta el 1927 se registraron 174 jamaiquinos naturalizados. ${ }^{33}$ Siguiéndole la pista al proceso de naturalización que debían seguir los descendientes jamaicanos, Diana Senior hace referencia al concepto de renaturalización utilizado en los años treintas:

"Lo que el Mr. Nesbeth y otras personas entendieron por re-naturalización fue el hecho de saberse costarricense pero no serlo legalmente, debido a la negligencia y/u omisión administrativa en la inscripción civil de los nacimientos, así como el descuido por parte de los progenitores; o porque al naturalizarse estos últimos, debían realizarle el mismo trámite a sus descendientes, sobre todo si eran menores de edad nacidos en el país”. ${ }^{34}$

Como se señaló antes, en las primeras décadas primaba en la comunidad el deseo de posesión espiritual de los danzantes. Chomsky, 180-200.

31 Entre los autores que comentan la falta de unidad dentro de la comunidad negra, están: Putnam, Lara. “La ideología racial, práctica social y Estado liberal en Costa Rica”, Revista de Historia (Costa Rica) 39 (enerojulio 1999), 171; Harpelle, quien hace importantes señalamientos sobre la división dentro de la comunidad negra. Véase: Harpelle, 188.

32 Las opciones son las diferentes leyes bajo las cuales se puede justificar la solicitud de naturalización. En el Registro Civil hay formularios para tramitar las naturalizaciones, con su respectiva ley y requisitos; por ejemplo: a) Ley 1916: para hijos de extranjeros nacidos en Costa Rica, mayores de 25 años; b) Ley 1902: para hijos de costarricenses nacidos en el exterior; c) Ley 1155: requisito para la naturalización por residencia oficial; d) Requisito para naturalización por matrimonio; e) Con fundamento en la ley 1902 para extranjeros que demuestren su estadía de 20 años en el país. Véase: Registro Civil, Sección de Opciones y Naturalizaciones, modelo de formularios originales y vigentes.

33 Ronald Quirós, "Otro significante en la...”, 22

34 Diana Senior, "La incorporación social en Costa Rica de la población afrocostarricense durante el siglo XX, 1927-1963” (tesis de Maestría en Historia UCR, 2007): 171-172. 
regresar a su patria y no se interesaba por lo nacional. Por lo tanto, más que rechazo a lo nacional hubo indiferencia. Posteriormente, al ser abandonados por esas patrias (Inglaterra y Jamaica) y segregados tanto por la sociedad nacional como por las compañías extranjeras, se gesta un cambio gradual de mentalidad en cuanto al sentido de pertenencia local. Poco a poco se convencieron de la importancia de establecerse, sobre todo considerando sus derechos por haber nacido en Costa Rica. Así que se asume la naturalización como la única forma posible para convertirse en costarricenses. Sobre las naturalizaciones para 1950, Diana Senior ofrece el siguiente dato:

“...para el censo de 1950 al menos 6.000 personas del total de "negros” en la provincia de Limón ya eran costarricenses, sin contar con los 1.369 “negros” repartidos en las restantes provincias del país...la mayoría de la población solicitante había nacido entre 1927 y 1933, de manera que la media en la edad para naturalizarse fue de 26, seguida de 27,30, 28 y 33; situación que refleja un segmento de la población económicamente activa en edad reproductiva y con deseo de lograr una estabilidad personal y familiar”. ${ }^{35}$

Estas cifras revelan un cambio de actitud de las nuevas generaciones, y su decisión de lograr un arraigo que les brinde estabilidad. Con la naturalización en sus manos, la población negra seguiría hasta la ciudadanía, proceso complejo y de continua redefinición.

Casi al final de la primera mitad del siglo XX, la Guerra Civil de 1948 no solo sorprendió a Costa Rica, sino a toda la región latinoamericana. Producto de este conflicto bélico y del cambio político que este trajo, las políticas estatales hacia la región caribeña cambian sustancialmente.

La nueva situación se hizo sentir el 4 de noviembre de 1949, cuando la Junta Gubernativa, precedida por José Figueres, firmó el decreto Ley n. ${ }^{\circ} 836$, el cual dejaba sin efecto el párrafo 30 del artículo 5 de la ley n. 31 del 10 de noviembre de 1934, que prohibía a los negros trabajar en el Pacífico. También se emitió una ley para derogar la discriminación racial y en favor del libre desplazamiento en la República. Además, se nombró una comisión especial para el empadronamiento de toda la población de color en Costa Rica, con miras a arreglar la documentación que los integrara al libre ejercicio de la ciudadanía costarricense.

\section{La compañía bananera como actor privado y empresarial}

La compañía bananera fue un actor importante en el entramado proceso de la construcción

35 Ibíd., 278-279. 
de las identidades de esta población. La literatura sobre la transnacional es abundante y diversa, pero nuestro interés es mostrar la relación de la compañía bananera con la población negra del Caribe costarricense, a fin de determinar si la estrategia de dicha compañía de mantener a los grupos étnicos separados influyó en la construcción de las identidades de esta población.

La United Fruit Company (UFCO) operaba en más de once países latinoamericanos ${ }^{36}$, entre ellos Costa Rica. Tal diversidad le permitió obligar a los Gobiernos a otorgarle incentivos. En Costa Rica, a la Compañía se le cedió del ferrocarril por 99 años y se le otorgaron facilidades portuarias en Limón y vastas extensiones de tierras (800 000 acres); además de llevar negocios de banano en forma paralela a la construcción del ferrocarril, obtuvo concesiones de 500 hectáreas por cada kilómetro de vía construida. ${ }^{37}$ La UFCO no solo fue el dueño absoluto de la producción de banano, sino también del transporte ferroviario, y tenía control y dominio en todos los aspectos de la vida de los trabajadores, pues el Estado se desentendió casi por completo de la región.

Un hecho importante que tendrá repercusiones futuras en la población negra es la temprana campesinización ${ }^{38}$ de la población. Para 1878, en la crisis del ferrocarril Minor Keith mantuvo a los trabajadores sin emigrar, gracias a un acuerdo al que había llegado en 1874 con el Gobierno de Costa Rica, para que diera tierra a sus trabajadores en la cual cultivaran productos de subsistencia. Se inició una rápida campesinización que tuvo sus repercusiones en una opción de consolidación colectiva, donde, sin duda, la tradición y la cultura caribeña de la familia extensa

36 La UFCO tenía subsidiarias que producían o compraban bananos en Costa Rica, Colombia, Ecuador, Panamá, Honduras, Belice, Guadalupe, Jamaica y Filipinas. Anteriormente la empresa también tuvo operaciones en Cuba, República Dominicana, Guatemala, Nicaragua y México. Véase: Bourgois, 51.

37 Brignoli Pérez, Breve historia contemporánea de Costa Rica, (México, FCE, 1997), 69.

38 La campesinización de la población negra ha sido abordada por diferentes autores: Bourgois, ofrece datos de un estudio realizado por Charles Koch donde muestra cómo los campesinos podían ganar treinta y un centavos por cada ocho centavos pagados a un trabajador corriente, y argumenta que fueron los obreros ferrocarrileros desempleados quienes originaron la producción bananera. Véase: Bourgois, 201. C. Murillo, al referirse al proceso de campesinización, plantea que esta se abre paralelamente al trabajo ferroviario y de plantación, y es enfática al señalar: que esta campesinización no facilita la participación a la vida nacional, ya que la mediana o pequeña finca tenida por los negros, más que proyectarse al mercado extrarregional, se constituye en una forma de estrategia de supervivencia y en un foco de resistencia cultural. Véase: Murillo, "Vaivén....”, 201. Tratando de rastrear el término, encontramos que, al analizar las ocupaciones de la población de Limón según el censo del 1927, Ronny Viales indica que quienes tuvieron mayor opción para incorporarse a los trabajos por "cuenta propia" en tierras ajenas fueron los negros. Por nuestra parte, pensamos que, además de iniciarse bastante temprano, la campesinización fue forzada por un momento de crisis. Interesa destacar en este trabajo que esta población no ha perdido ese vínculo con la tierra (lo que le ha permitido cierta autonomía y reproducir su cultura), llámese temprana campesinización, o forzosa, como proponemos, o por “cuenta propia” como también se la llama. 
se consolidaban. La población negra dentro de la Compañía logró ir escalando niveles medios, trabajos suaves, gracias al idioma, a la propagación de su "docilidad", a ser pequeños finqueros y solo emplearse a medio tiempo.

En el caso del Caribe costarricense, la UFCO usó una política de aprovechamiento de la diversidad étnica (indígenas, negros caribeños, centroamericanos, chinos) que ha sido objeto de estudio de varias investigaciones. ${ }^{39}$ Luego de varios experimentos, determinó que los jamaiquinos eran los trabajadores idóneos para el trabajo en la región, y procedió a su contratación masiva. Entre las cualidades valoradas para su contrato están: comparados con otros extranjeros, los jamaiquinos eran dóciles y toleraban el orden social que asignaba, según su fenotipo, una vivienda, un salario y un tratamiento inferior.

F. Bourgois, citando a Duncan y Fallas Monge, apunta que los jamaiquinos eran considerados dóciles, que no se inmiscuyeron en huelgas y que colaboraron y trabajaron gratis por ocho meses para el Sr. Keith cuando la Compañía enfrentó problemas económicos. Sin embargo, el mismo estudio muestra que un análisis más profundo del material disponible de las fuentes primarias, refuta el mito de su pasividad.

Hubo huelgas violentas, disturbios laborales e intentos de organizar sindicatos por parte de los trabajadores negros en las décadas de 1910 y 1920, acciones instrumentadas mediante la Artisan and Labourer Union. Una de ellas es la primera confrontación entre los trabajadores negros y la gerencia, documentada con fuentes primarias, ocurrida durante la construcción del ferrocarril cuando, según Fallas Monge, “algunos trabajadores negros pretendieron acometer con machetes a dos mandadores de raza blanca y los agredidos se vieron en necesidad de hacer usos de sus revólveres e imponer respeto y orden”. ${ }^{40}$

La UFCO no solo fomentó la división entre negros y latinos, sino entre los mismos caribeños. Definió diferencias jerárquicas, que variaban los grados de explotación del trabajador según su procedencia; por ejemplo, los trinitarios se catalogaban como perezosos; los jamaiquinos, con buen nivel educativo y respetuosos del orden, por lo cual se concentraron en trabajos suaves; 39 Bourgois, Philippe. Banano, Etnia y lucha social en Centro América. San José, Costa Rica: Departamento Ecuménico de Investigaciones, 1994; Chomsky, Aviva. West Indian Workers and the United Fruit Company in Costa Rica, 1870-1940. Banton Rouge: Louisiana State University Press, 1996, entre otros.

$40 \quad$ La misma fuente señala varias huelgas y manifestaciones protagonizadas por los negros, como la huelga violenta de 1913 en los distritos de Sixaola y Salamanca, y de nuevo la huelga más grande y violenta ocurrida en Sixaola en 1918, la cual duró tres meses. Véase: Bourgois, 96. 
y los caribeños francófonos se consideraban viciosos.

Por el gran descontento obrero acumulado, en 1934 estalló la huelga que movilizó a más de diez mil trabajadores de las plantaciones. A los líderes de esta huelga se los persiguió y arrestó, y enfrentaron procesos judiciales. La poca participación de los negros en esta huelga se atribuye a dos elementos: su condición de pequeños y medianos productores que se perjudicaban con la huelga, y su condición de extranjeros.

La transnacional sistematizó una estrategia de "dividir para vencer", basada en la manipulación de las diferencias étnicas. La fuerte animosidad racial entre los grupos étnicos (indígenas, centroamericanos y negros), promovida por la empresa, impidió desarrollar lazos de solidaridad entre los obreros. Una carta del secretario general a un dirigente del Partido Comunista en la huelga de 1934, ilustra el profundo conflicto: "La compañía ha estado fomentando la división entre negros y blancos, porque cuando los trabajadores están divididos son más débiles y no son capaces de luchar contra la compañía. Trate de seguir adelante y ganarse con cuidado cada día más negros hacia nuestra fila..."

Los intereses encontrados generados por las posiciones racistas de la empresa y los discursos clasistas de los sindicatos, se dirimen a favor de la empresa y el 7 de diciembre de 1934 se firma el contrato Cortés-Chittenden entre la UFCO y el Estado, donde se acuerda el traslado de la bananera al Pacífico Sur.

Con categorías étnicas, la UFCO mantuvo divididos a los trabajadores, los explotó y generó rechazo y división entre los negros, indígenas, centroamericanos y nacionales. Pese al esfuerzo de varios dirigentes, el partido comunista no pudo atraer el apoyo negro, y más bien hubo una mutua desconfianza que beneficiaba a la compañía bananera. Otra ventaja para la empresa con esta antipatía entre negros y mestizos, fue que las fuerzas gubernamentales podían movilizarse más fácilmente contra los negros, en especial considerando que, aunque los negros nacieran en el país, eran considerados extranjeros.

La táctica de "divide y vencerás” de la UFCO, mediante la introducción de distintas identidades étnicas o regionales de rompehuelgas, coartó el desarrollo de la solidaridad, indispensable para el movimiento laboral efectivo. Así, la empresa se convierte en un poderoso actor económico que utilizó la diversidad étnica de los trabajadores para explotarlos más, con lo 41 Bourgois, 103. 
cual fomentó el racismo entre los diversos grupos de trabajadores y, con ello, frenó la integración de este grupo étnico al resto de los trabajadores locales.

\section{Comunidad no negra ${ }^{42}$ como actor colectivo}

En las primeras décadas de las operaciones del ferrocarril y la compañía bananera, la población costarricense, inmigrante del resto del país, era muy exigua. La Compañía no pudo atraer a los costarricenses ni a los centroamericanos a trabajar en la inhóspita zona bananera; además, las condiciones de trabajo eran inferiores a las que estaban acostumbrados. Pero en 1872, en uno de los primeros informes sobre la obra del ferrocarril escrito por don Guillermo Nanne, gerente constructor del ferrocarril, se lee que de Pacuare al Reventazón "se han empleado peones costarricenses bajo la hábil y mágica dirección de don Francisco López Calleja. De la sección de Limón hasta Madre de Dios he mandado a retirar todos los peones por problemas de salud" ${ }^{43}$ Esta cita refleja cuán difícil resultaba para los costarricenses el clima de la región caribeña, sobre todo en las primeras décadas, por las precarias condiciones sanitarias y la nula experiencia en trabajos de construcción.

Fue lenta la incorporación de los costarricenses a la zona, pues antes del comienzo del siglo los salarios del Valle Central eran superiores a los de las bananeras. Los primeros en llegar a la zona masivamente fueron los nicaragüenses, empleados como rompehuelgas en $1913^{44}$, pero no permanecieron mucho tiempo por las condiciones de trabajo. Solo cuando mejoró el salario de las bananeras, llegaron grupos de guanacastecos y luego del Valle Central. En los años veintes el panorama cambió: la población negra perdía mayoría, por la creciente inmigración de trabajadores nicaragüenses y costarricenses.

Las relaciones interraciales entre los trabajadores fueron conflictivas. Según apuntan los diarios, a fines de 1906 se dio una huelga de trabajadores nacionales ocupados por la United Fruit Company en los muelles de Limón, la cual obedecía a que los trabajadores nacionales ganaban mucho menos que los afrocaribeños: mientras estos percibían un peso oro, aquellos recibían 85

42 Se usa la categoría “comunidad no negra” porque resulta más idóneo agrupar en ella a los costarricenses, sean del Valle Central o de Guanacaste, y a los centroamericanos. También encajarían en ella otras minorías étnicas no negras presentes en la región del Caribe. Optamos por “comunidad no negra” en vez de utilizar los términos de mestizos, hispanos, latinos, pañas, entre otros, por considerar que no hay unidad de criterios entre los estudios del tema sobre estos conceptos.

43 Gaceta Oficial, 11 de diciembre de 1972, 1, citado por Meléndez y Duncan, 62.

44 La información, 29 III. 1913:2. 
centavos. La situación se planteaba en los siguientes términos:

"Es sabido que el negro es mal trabajador, flojo por naturaleza, débil por temperamento [...] Y para afirmar que los trabajadores nacionales son superiores a los negros en el embarque de banano, basta decir que los primeros gastan a lo sumo cinco horas para el cargamento de un buque, mientras que los segundos no lo hacen en diez [...] el mal proviene de que, por razones de raza, los señores americanos de Limón, nos referimos a los que puedan tener parte en este asunto, no confraternizan con los nacionales”. ${ }^{45}$

Desde el inicio hubo conflictos con los negros, pues los costarricenses creían que debían obtener los mejores puestos y salarios; pero, por no tener experiencia ni dominio del inglés, la compañía prefería a los negros, lo cual irritaba mucho a los nacionales. Estos argumentaban desde la defensa de la soberanía nacional hasta la inferioridad étnica de los negros. Conforme se empezaban a sentir los efectos de la crisis de 1929, la Compañía y los negros eran el centro de ataque de los costarricenses, quienes demandaban del Gobierno que el 80\% de los empleados de la compañía fueran nacionales y se expulsara a los negros.

El resentimiento contra los negros se explicaba por las preferencias de la compañía hacia ellos, por su supuesta docilidad y por el idioma. Además, desde los años treintas, los trabajadores negros cambiaron su posición en la jerarquía laboral: rehusaban los trabajos penosos, y algunos se habían convertido en pequeños y medianos propietarios. En su percepción del “otro”, los nacionales no tomaban en cuenta las condiciones laborales de los negros ni el racismo contra estos, sino los beneficios; no entendían que si eran mayoría se debía a la alianza entre el Estado y la compañía. En 1933, más de 550 “blancos” exigieron la participación del Gobierno en lo que bautizaron “el problema negro”. La tensión se agravaba ante las amenazas de los negros, que eran mayoría y aducían estar armados. El temor motivó la petición para que el Estado interviniera ante el Congreso con el fin de que:

“... ponga remedio a esta situación humillante en nuestra propia patria por una raza inferior a la nuestra, que no tiene derecho a invadir nuestros campos, nuestras ciudades y nuestros hogares reduciéndonos a la miseria, porque están apoyados por una compañía que también ejerce un poder extraordinario en la provincia de Limón...”. ${ }^{46}$

45 “Huelga en Limón”. El Noticiero. Año V, n.o 1344 (S. J., 9/12/1906), p. 1.

$46 \quad$ ANCR serie Congreso n. ${ }^{\circ}$ 15753. f. 1v., citado por Viales, 158. 
La respuesta a esta petición fue el artículo 8 de la Contratación Bananera de 1934, el cual prohibía el trabajo a gente de color en la bananera del Pacífico Sur. La ley afectó profundamente a la comunidad negra, y enseguida 34 trabajadores negros, costarricenses por nacimiento y en ejercicio del sufragio protestaron ante los diputados Juan Romagosa y Virgilio Chavarri; argumentaban que el artículo 8 faltaba a la libertad de trabajo e incurría en un error por no contemplar que hay costarricenses naturales de color, y al final pedían un "exceptúense" y que se les permitiera trabajar en el Pacífico. La petición fue negada.

Con el contrato de traslado de la UFCO al Pacífico en 1934, el Estado recupera amplios territorios bananeros para asentar a inmigrantes del resto del país y ex obreros. A estos sitios llegan para quedarse inmigrantes nacionales procedentes de otras regiones del país. Las relaciones entre negros y mestizos no eran buenas; estaban permeadas por los prejuicios que cada grupo tenía sobre el otro. El color de la piel no era el único problema, pues la Compañía aprovechó el idioma, educación, religión, costumbres, aspiraciones y otros elementos para sobredimensionarlos y mantener divididos a los trabajadores. Si en el inicio los negros eran mayoría en la bananera y en la región, los no negros desplazaron poco a poco a los negros. Entre 1927 y 1950 había una clara división étnica: los negros predominaban entre la línea férrea ubicada entre Siquirres y Limón, y los nacionales en la zona de Línea Vieja. Las fricciones entre ambos grupos fueron permanentes.

\section{La comunidad negra como actor colectivo}

La situación de los inmigrantes jamaiquinos en la primera década fue muy difícil: un ambiente inhóspito, selva virgen, carencia de muchos de los servicios básicos, atención casi nula por parte del Estado; pero sí fueron efectivas las leyes racistas, la segregación y la exclusión a que los sometieron. A este panorama hay que agregar la estrategia de control de la empresa, basada en un trato racista de manipulación étnica hacia los diferentes grupos.

Ante esta situación, la comunidad negra comienza a verse diferente, a valorar su cultura y a establecer diferencias entre ellos y los diversos "otros" (costarricenses, norteamericanos, indígenas, negros francófonos, centroamericanos y chinos). Por la barrera idiomática, solo se pueden comunicar con los norteamericanos o ingleses, con quienes comparten rasgos de la cultura británica pero reciben un trato extremadamente racista. Con respecto a la costarricense y demás culturas, se sienten más educados; y ante el abierto rechazo de estos hacia ellos, no se interesaron 
en relacionarse. Desde este momento en que se sienten diferentes de los "otros", perciben que esos "otros” también los ven distintos y les asignan roles, etiquetas y estatus que ellos rechazan o asumen. Entonces se empiezan a gestar — de forma consciente o no— sus posibles estrategias identitarias.

\section{Estrategias identitarias: familia, iglesia y escuela}

Las acciones de la comunidad negra se dirigían, principalmente, a mantener su cultura viviendo en comunidad y sin mezclarse con los “otros”. Su cultura descansó en tres pilares: familia, iglesia y escuela. Desde su llegada se crearon pequeñas escuelas en inglés, financiadas por la comunidad; cuando llegan el primer misionero bautista, los metodistas y anglicanos, se popularizan las escuelas parroquiales. ${ }^{47}$ Entre las escuelas y las iglesias hubo una colaboración estrecha; en muchos casos las iglesias formaban o apadrinaban las escuelas, y desde estas se promovía la lectura de la Biblia.

Para 1927 había en Limón 33 escuelas privadas a las cuales concurrían 15000 alumnos ${ }^{48}$, en su mayoría de origen jamaiquino. Estas escuelas funcionaban con maestros traídos desde Jamaica y con textos británicos. Se realizaron grandes esfuerzos por mantener estas escuelas, y en muchos casos no les interesaba que sus hijos asistieran a las escuelas en español, pues esperaban regresar a su patria. Por el activismo protestante, que conlleva la necesidad de leer la Biblia, esta comunidad tuvo un elevado nivel de alfabetización frente a otros grupos, y esto se ha usado como una estrategia en la construcción de sus identidades.

Se señala que, con el funcionamiento de las escuelas estatales a partir de 1922, surgieron distinciones entre los diferentes grupos; al mismo tiempo, estas fueron un terreno de interacción de las diferencias culturales entre negros y no negros, mientras las escuelas eclesiales de inglés fueron siempre un espacio para la reproducción de rasgos de la identidad que fundaban solidaridades étnicas. Muchos miembros de la comunidad de hoy consideran que la religión fue y es el más poderoso transmisor de los valores esenciales de la cultura negra; era un altar de disciplina, todo lo que se enseñaba en ese entonces venía de la iglesia; no solo se enseñaba la

$47 \quad$ El 27 de mayo de 1888 llega el primer misionero protestante: el reverendo José Heat Sooly; en 1897, un pastor anglicano de El Salvador; entre el 1901 y 1903, los metodistas; el Ejercito de Salvación llega en 1907, y los adventistas arribaron alrededor de 1915. Véase: Luchas y esperanzas: 100 años de historia doble e inconclusa del cantón de Limón (Limón, Costa Rica: Municipalidad de Limón, 1992), 270.

$48 \quad$ Meléndez y Duncan, 78. 
Biblia, sino también lo relativo a la buena conducta; se sancionaba y dirigía la vida de la gente. Por eso la iglesia era el centro de operaciones en el plano mental y espiritual de la vida, como lo señala el testimonio siguiente:

"De los elementos identitarios que caracterizan a los primeros inmigrantes jamaiquinos está en primer lugar la espiritualidad en diferentes formas. En cada barrio había escuelitas parroquiales en inglés, también había escuelitas particulares. Esas escuelitas no dejaban dinero, pero formaban con rectitud y gran temple a los muchachos. Hoy hay varias iglesias que tienen escuelas pero no con ese sentido cultural y de servicios que tenían antes, sino como empresas que dejan dinero; son una oferta más”. ${ }^{9}$

Este testimonio se corrobora con varios de los periódicos de la época, que reflejan la importancia de la vida espiritual en la comunidad y de las escuelas eclesiales. ${ }^{50}$

Además de los elementos citados, las logias tuvieron un papel relevante en esta comunidad. Estaban fuertemente arraigadas y a ellas pertenecían las personas más respetadas; sus ritos de iniciación eran verdaderas pruebas de valor. Se caracterizaron por la hermandad y la fraternidad entre sus miembros. Anualmente tenían fiestas y ceremonias de gran vistosidad, que servían como punto de reunión de la comunidad; entre estas estaban: el día de la emancipación, el día del rey, y después el día de la reina de Inglaterra, lo cual evidencia su condición de súbditos británicos. Este tipo de organización fue otro medio de visualizar socialmente al grupo negro, y en Limón hubo hasta 40 logias. ${ }^{51}$ La comunidad también tenía buenos deportistas, y se destacó en cricket, futbol, béisbol, boxeo y atletismo.

Otro elemento que identifica a este grupo con su espacio físico es la toponimia de la región; basta pasearse por cualquier lugar para observar que los nombres de los lugares hacen referencia a Jamaica, Gran Bretaña y en algunos casos a África. ${ }^{52}$ La campesinización temprana de la

$49 \quad$ Entrevista a la Sra. Marlene Dell Hamilton, pastora de la Primera Iglesia Bautista de Limón. Universidad Bíblica Latinoamericana, San José, 9 de noviembre de 2006. La Sra. Dell es la primera mujer reverenda de la iglesia bautista de Limón. La primera iglesia bautista de Jamaica en Costa Rica se fundó en 1888, y en su época fue la segunda iglesia protestante y la primera iglesia bautista fundada en Costa Rica.

$50 \quad$ Heraldo de Limón, 1909; Heraldo del Atlántico, 1911; y El pueblo limonense, 1915.

51 Según relata Ana Cristina Rossi, en un artículo llamado "Entre vestigios", donde comenta una entrevista realizada a Alfred Henry King, para la primera mitad del siglo XX, según apreciaciones del Sr. King y del conteo de los periódicos de la época hecho por Ana Cristina en Limón existían más de 40 logias, para una población de cincuenta mil negros.

52 Lugares céntricos de Limón, llamados: Jamaica Town, hoy barrio Roosevelt; Nuevo Gales; New Castle 
población le permitió, además de estar aislada, la libertad de conservar sus hábitos alimenticios al cosechar los tubérculos caribeños de su dieta.

La música jamaiquina es uno de los principales rasgos de la comunidad negra. La música es parte fundamental de la vida de todos los afrocaribeños, y los primeros inmigrantes trajeron a Costa Rica sus instrumentos, pues una gran parte de los maestros de inglés también enseñaba música. La iglesia jugó un papel crucial para los inmigrantes, y también fomentaba el estudio de música de instrumentos. En cada iglesia se tocaba órgano y otros instrumentos; había tantos músicos en las iglesias, que se turnaban para poder tocar, y era muy común que salieran a tocar a las calles, para atraer a las personas..$^{53}$

El mento jamaiquino, el calipso, los cantos religiosos, el baile de cuadrilla y el uso de instrumentos de origen africano y caribeño, son algunos ejemplos de la rica variedad de la expresión cultural de Limón. Se destacan los cantos denominados calipsos, cuya permanencia y popularidad data por lo menos de la década de $1940 .{ }^{54}$ Esta música ha marcado el paisaje limonense y ha generado un repertorio propio, que refleja la vida y la historia de la comunidad negra. Al establecerse un nexo de identidad entre la producción sonora y el grupo étnico, se puede considerar al calipso una expresión musical de la cultura negra limonense que conscientemente ha sido cultivada por esta población y que, en el proceso, ha devenido en música étnica ${ }^{55}$, constituyendo así en uno de los rasgos culturales seleccionados por esta comunidad para resaltar las diferencias étnicas.

\section{Influencia de los movimientos panafricanistas en Limón}

En la década de 1920, un jamaicano que causó gran impacto en la población negra de Limón fue Marcus Garvey. Su pensamiento y obra caló tan fuerte en esta población, que aún perdura. La situación de los inmigrantes negros antes de que llegara M. Garvey con sus ideas de unidad, dignidad, respeto y grandeza para la raza negra, era muy difícil. A partir de 1910, esta población vive un proceso marcado por una serie de conflictos económicos y raciales con la empresa bananera y con la población mestiza, los cuales evidencian las diversas formas de lucha adoptadas por esta población. Esto contradice la idea difundida sobre la pasividad y conformismo

y Glasgow, Liverpool, entre muchos otros.

53 Municipalidad de Limón: Luchas y esperanzas, 340.

54 Manuel Monestel, Ritmo, canción e identidad (San José, Costa Rica, Euned, 2005): 27.

55 Ibíd. 
de los negros. ${ }^{56}$ Desde ese momento la población costarricense, como producto de la división fomentada por la empresa, solicita al Estado costarricense la expulsión de inmigrantes negros, por cuanto estos ocupan los puestos de trabajos que pueden ser desempeña por los costarricenses. ${ }^{57}$

Los inmigrantes negros sienten como nunca antes la explotación de la empresa y el racismo y rechazo de los costarricenses. En esta situación de desesperanza aparece el mensaje de Marcus Garvey, con su propuesta de dignidad y unidad de la raza negra. Garvey había vivido y trabajado en Limón entre 1910 y 1911, por lo cual conocía desde dentro las condiciones de las bananeras. La comunidad recordaba su intento de organizar un sindicato; esto le costó su despido, y le dio ante la comunidad un gran prestigio y credibilidad.

En 1914, Garvey funda en Jamaica la Universal Negro Improvenment Association (UNIA). Su mensaje enfatizaba que era hora de que los negros se desarrollaran por sí mismos y se convirtieran en hombres y mujeres de alta moral, y la UNIA era el camino para lograr ese objetivo. Para ello se conformó una red de centros como puntos de encuentros, orientación y organización, llamados Liberty Halls, en los cuales funcionaban cooperativas de asesoramiento económico para negocios de la población negra.

En 1922 se abre en la ciudad de Limón el primer Liberty Halls. Más adelante se abren filiales en Siquirres, Matina, Madre de Dios, Cahuita y Puerto Viejo, y llegan a funcionar 21 filiales, testimonios del gran impacto de las ideas de Garvey. Indudablemente el discurso de Garvey unió a la población en torno a sus raíces africanas y a la posibilidad de construir un proyecto propio, marcando con ello un elemento importante en la construcción identitaria.

$56 \quad$ En la década de los veintes, la población negra participa en una serie de luchas por reivindicaciones, donde se destacan: en 1910 se formó un sindicato de artesanos compuesto casi exclusivamente por negros (Jamaica Times, 12, III; 1910:1). En respuesta, la empresa bananera comenzó a despedir a los supervisores negros miembros del sindicato. Otro hecho importante fue la huelga de 1913 en los distritos de Sixaola y Salamanca, en la cual la empresa contrató a 200 nicaragüenses para sustituir a los negros, lo cual provocó grandes disturbios. Para más detalles, véase: La información, 25.III1913:5; 26. En 1918, en Sixaola hubo otra gran huelga de los obreros negros, que duró tres meses, en la cual demandaban aumento salarial.

57 Varios autores se refieren a esta solicitud. Patricia Alvarenga, citando a Jeffrey Casey, señala que ya en 1920 la compañía bananera estaba en una situación difícil y los empleos disminuyeron significativamente; pero los discursos discriminatorios entre los trabajadores blancos toman fuerza en medio de la crisis del 1930 . En esta, los trabajadores blancos hacen dos solicitudes: 1) erradicar hasta donde sea posible de la mano de obra negra, para impedir toda inmigración y realizar deportaciones masivas; y 2) impedir a los negros realizar labores especializadas. Alvarenga señala que, en este contexto, en 1932 el Congreso aprobó por amplio margen una propuesta de Otilio Ulate para prohibir a los negros participar en los nuevos bananales del Pacífico. Véase: Alvarenga, 10. 
Los limonenses recuerdan que, en los años treintas, todo Limón se paralizó al reunirse 18000 personas a escuchar a Garvey en un acto público. ${ }^{58}$

Otro elemento relevante aportado por el garveyismo a la construcción identitaria, es la estrecha relación que propicia en toda la región del Caribe, incluyendo islas hispanohablantes y francófonas, y en especial con Estados Unidos, sede central del movimiento. La UNIA contaba con filiales en cuarenta países y dos millones de miembros contribuyentes. Inspiraba a los negros a rechazar el complejo psicológico de una autoestima lastimada y destacaba los valores y dignidad de su cultura, por lo cual pronto representó una amenaza para los países y empresarios que fomentaban el racismo y la explotación basada en la diferencia étnica. La represión contra el movimiento fue violenta y sistemática, y la Compañía comenzó a despedir a los miembros de la UNIA en Limón; en Estados Unidos se desacreditó y encarceló a su líder, acusándolo de usar el correo de ese país para vender acciones del Black Star Line cuando la compañía ya no era solvente. ${ }^{59}$

Ya en los años cuarentas, el garveyismo había decaído internacionalmente. Aunque con muy pocos miembros, hoy en Limón, a los 85 años de su fundación, la UNIA sigue activa, con el nombre de Black Star Line, el cual mantiene en lo fundamental la filosofía garveyista ${ }^{60}$ En 1988, gracias a las gestiones de líderes de la comunidad, el Ministerio de Cultura y Deportes declaró el inmueble como patrimonio histórico cultural y se ha remozado. Actualmente, la UNIA tiene como objetivo revivir la organización interesando e involucrando a la población joven. ${ }^{61}$

Como resultado de las luchas garveyistas, se pueden señalar lo siguiente: la población ha asumido con orgullo y dignidad las raíces africanas; se ha afianzado el sentido de solidaridad y pertenencia a la gran familia de la diáspora africana; se ha desarrollado en esta población la conciencia de luchar por una serie de reivindicaciones que trascienden lo económico, como la ciudadanía. Es decir, se puede identificar el avance cualitativo que ha significado el paso de esta población que luchó en la Asociación Universal para la Superación del Negro (UNIA) para $58 \quad$ En diferentes periódicos de la época se publicaron varias fotografías del acto. En la revista Limón Roots: edición n. ${ }^{\circ}$ 1, año 1, 1999, se reproduce una foto panorámica de actividad de la UNIA celebrada en 1926, plaza Yglesias, ciudad de Limón (hoy estadio Juan Gobán).

59 Milton Franklin, Marcus Garvey y el concepto del garveyismo (San José, Costa Rica, 1987), 35.

60 El edificio se mantiene en el centro de la ciudad, como testimonio de que la comunidad aún esta en pie de lucha por el rescate y revitalización de su cultura.

61 Entrevista a Margaret Simpson Chamber, directora regional de la biblioteca de Limón y miembro de la directiva de la UNIA en Limón. 2 de diciembre de 2006. 
convertirse en ciudadanos costarricenses. ${ }^{62}$

En pleno 1948, cuando tuvo lugar el mayor acontecimiento bélico de la nación, conocido como la Guerra Civil de 1948, la comunidad negra todavía era considerada extranjera, y esto fue determinante para que se mantuviera al margen de dicho conflicto. Lo interesante es que, en ese mismo año, dentro de la comunidad negra se organizaba lo que hoy es la manifestación cultural más afianzada en la comunidad: el carnaval limonense.

El activista comunitario, Alfred King, con un grupo de entusiastas amigos, organizó en 1949 el primer carnaval limonense. Logró involucrar y entusiasmar a la comunidad, que lo costeaba con sus propios recursos. Esta primera entrega tenía comparsas, disfraces, desfiles, buena música y, lo más importante: puso a la gente a bailar en las calles.

El objetivo era que la municipalidad recibiera las ganancias y las repartiera entre las instituciones. El carnaval se ha consolidado, y año tras año irrumpe como una afirmación de la cultura negra. Hoy su acogida es grande, pues ya no es solo de la comunidad negra, sino que se integran y participan masivamente las diversas etnias de la región. Se considera un valioso aporte de la comunidad negra a la provincia de Limón y al país.

\section{Los “otros” según determinados contextos}

Desde antes de la invención de la nación costarricense, el Estado ha tratado de consolidar un perfil de país homogéneamente blanco. Para mantener esa imagen, se han promulgado leyes migratorias donde se prohíbe la entrada a individuos considerados de raza inferior. Con estas leyes, el Estado busca preservar la pureza de la raza blanca de Costa Rica, en defensa de la identidad nacional.

La llegada y asentamiento de los inmigrantes jamaiquinos fue tolerado como un mal necesario, y coincidió con una época de un nacionalismo profundamente racista. Desde el inicio, los jamaicanos fueron vistos como los “otros”, que constituían un perfecto contraste con el discurso oficial. Los inmigrantes eran el "otro amenazante" 63 , que podía contaminar la pureza

$62 \quad$ Solo hasta el año 1949, con las reformas de la Segunda República, se le reconoce a esta población la ciudadanía costarricense. Véase. Senior, Diana. "La incorporación social en Costa Rica de la población afrocostarricense durante el siglo XX, 1927-1963”. Tesis de maestría en Historia, SEP, Universidad de Costa Rica, 2007. También, Molina Iván y Palmer Steven. Historia de Costa Rica. Breve actualizada y con ilustraciones (San José, Editorial de la Universidad de Costa Rica. 2004), 81.

63 El concepto de "otro amenazante" lo utiliza Carlos Sandoval en su libro: Otros amenazantes. 
de la raza blanca del pueblo costarricense. Para la sociedad costarricense el negro era un "otro", como lo eran también los chinos y los indígenas.

Para los inmigrantes jamaiquinos, el “otro” variará de acuerdo con determinados contextos. En las primeras décadas de la inmigración, los "otros significantes" ${ }^{4}$ eran los norteamericanos, de quienes dependían y quienes los sometieron a una explotación laboral racista. La sociedad costarricense y su rechazo, no le importaban mucho al jamaicano, pues el contacto era mínimo y la barrera idiomática contribuía a ello; más bien, su objetivo era ahorrar dinero y regresar a Jamaica. Tenían una mentalidad de retorno que se manifestaba en diferentes formas; por ejemplo: no pintaban sus viviendas; y no estaban interesados en que sus hijos aprendieran español, idioma que sería innecesario en Jamaica.

A partir de la llegada masiva de los nacionales a la zona y los conflictos cotidianos, el roce causa que el "otro significante" pase a ser el costarricense, paña, mestizo, blanco, vallecentralino o tico. Ambos grupos étnicos, negros y nacionales radicados en Limón, tenían prejuicios sobre el "otro". Los nacionales eran catalogados por los jamaiquinos como débiles, incapaces de construir su propio ferrocarril, hipócritas, perezosos, con poca higiene; mientras los nacionales calificaban a los negros como argollas, complacientes con los jefes, sumisos, alegres, bulliciosos, bailadores y estudiosos. ${ }^{65}$

La visión del "otro" podía variar según las circunstancias y los intereses políticos. A veces los negros jamaiquinos podían ser catalogados como pasivos, trabajadores y dóciles, como lo quería hacer ver la compañía bananera. En otras ocasiones se establecían diferencias según las procedencias: los jamaiquinos son "respetuosos del orden establecido", y los de Barbados propensos al "robo y pillaje".

Entre las diferentes denominaciones que ha recibido esta población, en un primer

Los nicaragüenses y la formación de identidades nacionales en Costa Rica (San José, Costa Rica: Editorial Universidad de Costa Rica, 2003). En nuestro caso, se emplea por considerar que los inmigrantes negros jamaiquinos eran vistos como una verdadera amenaza; su presencia se veía como una amenaza que podía contaminar la pureza de la raza blanca predominante en el Valle Central.

64 El concepto de "otro significante" lo usa Ronald Soto al referirse al inmigrante afrocaribeño en su estudio: "Un otro significante en la identidad nacional costarricense: el caso del inmigrante afrocaribeño, 18721926”. Para nosotros, este concepto le da importancia a un "otro"; en el caso de la población estudiada, variará según el contexto; se desea señalar que, para la comunidad jamaiquina, el otro no siempre fue el costarricense. 65 Estereotipos o percepción del otro que aparecen en los estudios de: Putnam, Bourgois, Harpelle, Soto Quirós, Chomsky y Duncan, trabajos ya citados anteriormente. 
momento prevalece el referente británico-jamaiquino como aglutinador étnico de esta comunidad. Ellos se denominaban a sí mismos como: súbditos-británicos, jamaiquinos-británicos, británicos, jamaiquinos, súbdito-real, ciudadano-británico. Los “otros” los denominaban: “negros jamaiquinos”, africano-británicos, negros británicos, antillanos, caribeño-ingleses, chumecos (forma peyorativa utilizada por los costarricenses, originada de la pronunciación hispanizada del vocablo inglés Jamaican). En un segundo momento, a partir de la década de los años veintes, la influencia de los movimientos panafricanistas se refleja en las autodenominaciones siguientes: jamaiquino-africano, africano, negro-africano, negro-caribeño, africano-caribeño, negro-limonense. Se identifica una redefinición de la identidad étnica basada en la asunción de la negritud, del panafricanismo.

\section{Conclusión}

Los inmigrantes jamaiquinos que llegaron en 1872 a la zona inhóspita del Caribe costarricense, vivieron un proceso sumamente complejo, el cual se ha analizado por el papel que jugaron los diferentes actores. El Estado se caracterizó por aplicar políticas racistas, de exclusión, y por facilitar la entrada del capital norteamericano a la región. Como se mostró, todos los esfuerzos estatales para atraer a una inmigración blanca fracasaron, y fueron precisamente los inmigrantes menos deseados quienes contribuyeron a poblar la zona, construir el ferrocarril y desarrollar la industria bananera. En este periodo, los negros (junto a los chinos) eran particularmente los "otros", que representaron un rol importante en la afirmación de la identidad costarricense, y viceversa; también los costarricenses fueron determinantes para la afirmación de las identidades de la comunidad de origen jamaiquino.

El segundo actor, la compañía bananera, controló por completo la vida de los inmigrantes jamaiquinos, utilizando estrategias de control laboral basadas en las diferencias étnicas. Con ello logró mantener divididos a los trabajadores y someterlos a la más brutal de las explotaciones económicas y racistas.

Un tercer actor, la comunidad no negra, formada sobre todo por costarricenses, interiorizó el discurso racista del Estado y lo usó en defensa de sus intereses, al percibir que los "otros" representaban una competencia en las bananeras. Esta situación se explica porque, desde la década de 1860 hasta la de 1940, el Estado desarrolló un racismo oficial, con leyes y discursos discriminatorios contra las “razas inferiores”. Esas jerarquías étnicas, junto al mensaje de la pureza 
de la raza nacional, calaron en una buena parte de la sociedad costarricense. Si bien las leyes cambiaron a partir de 1949, estas visiones persisten en un sector de la población costarricense. Así, el Estado, la UFCO y la comunidad no negra fueron actores negativos para la integración de la comunidad de origen jamaiquino a la nación.

El cuarto actor, la comunidad negra, en este proceso tan complejo no solo logra sobrevivir, sino también recrear sus identidades con estrategias identitarias que le posiblitan conservar su cultura y construir dos identidades a lo largo de este período. Desde el inicio, la comunidad construye un discurso de rechazo a la integración y elabora estrategias identitarias con base en la "negación" de los roles asignados. Se siente y se ve diferente, toma conciencia de ello, se aferra a su historia, revalora su cultura y lucha por mantenerla. Sus estrategias identitarias estuvieron basadas en lo que se puede considerar los tres pilares fundamentales de la cultura: familia, iglesia y escuela. A través de ellos se reproduce en la medida de lo posible su forma de vida (escuelas, iglesias, logias, organizaciones sociales, culturales, alimentación, vivienda, vestidos, música, bailes). Así, esta primera generación se caracterizó por ser angloparlante, protestante y súbdita de la corona británica, y por construir una identidad étnica con base en su condición de británicosjamaiquinos.

La segunda generación, a partir de la década de los años veintes, recibió un gran impacto de las ideas de los movimientos de la negritud ${ }^{66} \mathrm{y}$ las posteriores acciones y discursos de Marcus Garvey. Una gran parte de esa población construyó discursos y prácticas de identidad étnica donde se valoraban las raíces africanas y la pertenencia a la gran comunidad panafricana.

$66 \quad$ Antes de que se desarrollara el garveyismo, tanto en la región del Caribe como en Estados Unidos se conocían las luchas de muchos panafricanistas, entre ellos el haitiano Benito Silvan, quien en 1905 fundó una organización llamada The Universal Association for the Moral Improvement of Mankid (Asociación Universal para el Avance Moral de la Raza Humana). Otra organización que funcionaba en el mismo periodo fue "Los negros leales universales", dirigida por el panafricanista panameño James B. Yearwood en la zona del Canal de Panamá, entre otros. Véase: Milton Franklin, 14. 